\title{
Robust adaptive distributed beamforming for energy-efficient network flooding
}

\author{
Abdelrahman Abdelkader ${ }^{*}$ (D) and Eduard Jorswieck
}

\begin{abstract}
In a device-to-device wireless multi-hop communication scenario with resource-constrained devices, the energy efficiency of communication plays a significant role in network design and operation. Based on the recently proposed Glossy network flooding approach, we introduce cooperation between transmitting nodes and formulate a robust multicast beamforming problem with imperfect channel state information (CSI) and analyze its performance. The level of cooperation is dependent on the number of limited feedback bits from receivers to transmitters. First, the impact of the number of limited feedback bits $B$ on energy efficiency is studied, and the programming problem for finding the optimal $B$ is formulated subject to a maximum outage constraint of $5 \%$. Numerical simulations show that there exists an optimal number of feedback bits that maximizes energy efficiency. Second, the effect of the number of cooperating transmitters on energy efficiency is investigated. Results show that an optimum group of cooperating transmit nodes, also known as a transmit coalition, can be formed in order to maximize energy efficiency. Results show that the investigated techniques including optimum feedback bits and transmit coalition formation can achieve a 100\% increase in energy efficiency when compared to the state-of-the-art Glossy under the same operation requirements in very dense networks.
\end{abstract}

Keywords: Network flooding, Distributed beamforming, Limited feedback, Energy efficiency

\section{Introduction}

Vastly distributed sensors are becoming an important part of our daily lives where they are utilized in many applications and diverse fields such as smart homes, environmental monitoring, emergency, and health [1,2]. Furthermore, recent $5 \mathrm{G}$ initiatives show a clear focus on energy-efficient smart cooperative transmission protocols [3]. These initiatives are partially motivated by the importance of energy efficiency in large-scale wireless networks for the Internet of Things (IoT) and cyber-physical systems applications [4]. The devices used in these applications are usually heavily distributed, which makes them very challenging to be replaced or recharged in a frequent manner. Therefore, extending their battery life becomes crucial. Optimizing energy will help extend the expected lifetime of these small battery-operated devices and facilitate their large-scale deployment.

To this end, we extend our development in [5] where a device-to-device wireless multi-hop communication

*Correspondence: abdelrahman.abdelkader@tu-dresden.de Communications Laboratory, Faculty of Electrical and Computer Engineering, TU Dresden, Germany scenario with resource-constrained devices that require energy-efficient connectivity is considered. Based on the recently proposed Glossy network flooding protocol [6], that follows the IEEE 802.15.4 low-rate wireless personal area networks (LR-WPANs) standard, the beamforming and power control problem is formulated, analyzed, and assessed in network scenarios where the same information needs to be shared with all nodes. Performance measures are compared, where the use of beamforming has proven to improve energy efficiency significantly.

In this work, we move towards a more realistic scenario where the cost of cooperation in terms of energy consumption is taken into account. This cost of cooperation is represented by the energy consumed in the process of channel state information (CSI) feedback from receiving to transmitting nodes. We note that there are several other factors that contribute to the processing overhead of collaborative distributed multicast beamforming [7] in actual implementations, but for the scope of this paper, we focus on the theoretical analysis of the proposed approach. We leave the physical implementation impairments for future 
Table 1 List of abbreviations

\begin{tabular}{ll}
\hline CSI & channel state information \\
IOT & internet of things \\
LR-WPANs & low rate wireless personal access networks \\
MTC & machine type communication \\
D2D & device to device \\
DCBF & distributed collaborative beamforming \\
FOS & flooding overlay structure \\
MMSE & minimum mean square error \\
SIC & successive interference cancellation \\
CQI & channel quality information \\
QOS & quality of service \\
GCF & greedy coalition formation \\
\hline
\end{tabular}

research. This results in a trade-off between implementation complexity and energy efficiency [8]. Moreover, we analyze the consequence of changing the number of cooperating nodes on energy efficiency. We use the term coalition throughout the paper to address a number of transmitters that cooperate in order to perform multicast beamforming during any intermediate stage of a Glossy flood. We describe and analyze the performance of an algorithm that maximizes energy efficiency in cooperative network flooding by forming coalitions of transmit nodes.

After describing the system model in Section 2.1, we explain the proposed CSI feedback approach in Section 2.2, then we introduce our problem statement in Section 2.3. Following that, we formulate in Section 3 our general robust multicast beamforming problem under limited feedback, building therefore upon the results presented in [5]. Then, we analyze the effect of increasing feedback bits $B$ and optimal coalition size to maximize energy efficiency in Sections 4 and 5, respectively. We support this analysis with simulation results presented and discussed in Section 6, where we illustrate how the robust beamforming problem can be solved for application- specific requirements. Finally, we present our conclusions and an agenda for future works in Section 7. A list of regularly used abbreviations throughout the paper is shown in Table 1.

\subsection{Recent results on cooperation and beamforming in wireless sensor networks}

Cooperation in wireless networks is a topic that has attracted the attention of researchers for many years [9-12]. Distributed and collaborative beamforming is defined as a technique where independent and randomly located source nodes cooperate to transmit a common radio frequency signal, hence forming a virtual antenna array [13]. This cooperation improves the lifetime of the sensor nodes [14], proving to be valuable for systems with limited battery life. It also shines as a promising technique to empower many $5 \mathrm{G}$ technologies such as machine-type communication (MTC), device-to-device communication (D2D), and Internet of Things (IoT).

Initially, research on collaborative distributed beamforming in sensor networks was divided into two directions [15]: collaborative and distributed. On the one hand, collaborative beamforming focused on beam pattern analysis while assuming a perfect phase and carrier synchronization between sensor nodes [16]. On the other hand, distributed beamforming focused on the feasibility of achieving synchronization between those nodes [17]. However, some work was done in order to explore the possibility of achieving cooperation without synchronization such as in [18]. Later on, both directions started merging forming distributed collaborative beamforming. The recent survey [15] classifies research trends in this area very clearly, from which Fig. 1 is adapted. This classification indicates that our current work, where we maximize energy efficiency in network flooding by applying distributed collaborative beamforming (DCBF), lies within the scope of power minimization and lifetime maximization, more precisely under energy efficiency maximization.

Previous research efforts in this direction have several performance metrics for the evaluation of DCBF. Network

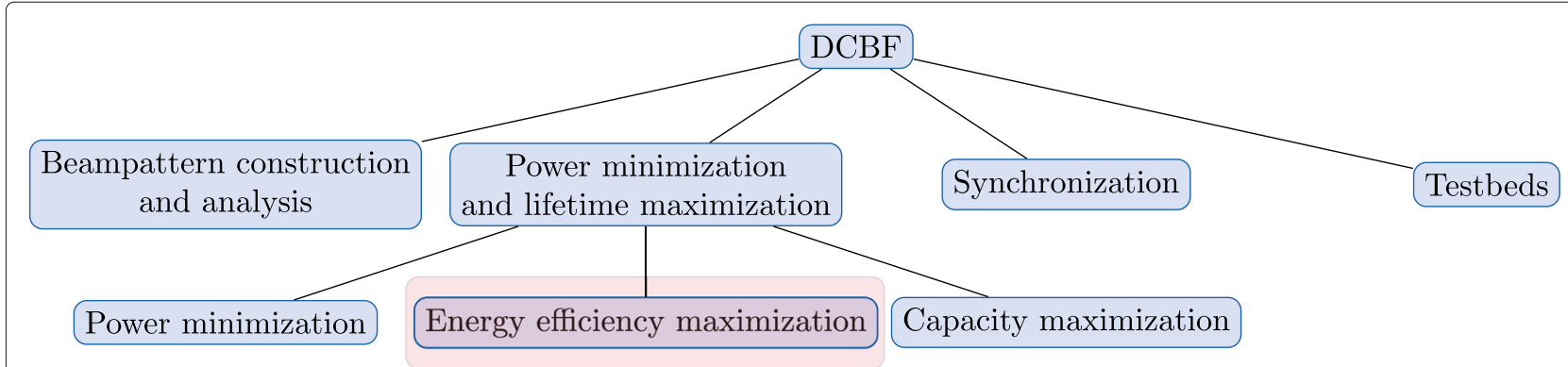

Fig. 1 Research trends. Research directions in DCBF in wireless networks 
lifetime is usually defined as the time duration from the start of network operations till the first node runs out of battery [14]. In [19], a power allocation algorithm using convex optimization is used to maximize network lifetime. On the other hand, total power consumption is defined as the sum of the total consumed power of each node in transmit, receive, and idle phases of operation [6]. In some works, e.g., [14], only transmit power is considered in the total power consumption. In [20], an algorithm using a dual problem convex optimization is proposed to minimize the total power consumption under individual power constraints. Another approach by [14] using genetic algorithm is proposed to minimize the transmit power consumption and increase the network lifetime. Moreover, maximizing energy efficieny is the goal of [21] where sequential quadratic programming is used to solve the problem of optimal node allocation; they also show the effects on spectral efficiency. Robust distributed beamforming is considered in [22], where beamforming is used to minimize the total relay transmit power under signal-to-noise ratio constraints with the availability of perfect channel state information. An effort to investigate distributed beamforming under limited feedback in cooperative networks is made in [23], where an optimal beamforming vector is proposed to maximize the received signal-to-noise ratio at the receiver. Although only a fixed number of feedback bits is considered, the results prove that this scheme is indeed beneficial in terms of performance.

\subsection{Network flooding state of the art}

Network flooding (also commonly known as data dissemination) is used in many applications ranging from environmental monitoring to industrial automation. For many of these applications, the main research focus is reliability and energy efficiency because of the limited battery lifetime of the sensor nodes. Many network flooding approaches are presented in the literature over the years. The simplest form of it is called Simple Flooding, where each node that receives a new message forwards it to a neighbor node. However, this results in excessive redundancy and network congestion [24]. A method to treat this problem is presented in [25], where a flooding overlay structure (FOS) is proposed to increase the network lifetime and reduce the overhead of flooding in a sensor network. Another example is Trickle, a network flooding algorithm for code propagation in wireless sensor networks [26]. Trickle uses an individual timer at each node to periodically send out a summary to the neighboring nodes but obligates the node to stay silent in case of receiving information similar to its own. Unfortunately, Trickle is not suitable for real-time applications due to the long time until stable operation which can go up to a few minutes. For real-time applications, Glossy is introduced in [6] to exploit constructive interference at the receiving nodes resulting from concurrent transmissions of identical packets form multiple nodes. For the successful use of concurrent transmissions, a high level of synchronization is needed, which is provided by Glossy down to $0.5 \mu \mathrm{s}$ for an eight-hop network. On top of a very simple algorithm, Glossy achieves very high reliability with low latency. However, scheduling individual floods for each source node can cause a delay until all data is disseminated successfully. To remedy this, MFGlossy is proposed in [27], where physical layer network coding enables multiple simultaneous floods in Glossy. The results show increased energy efficiency and goodput when compared to the standard Glossy on the expense of a complicated receiver design.

A Glossy flood typically starts with one node transmitting certain information at maximum transmit power $(0 \mathrm{dbm})$. Then, all nodes, which receive successfully and have not reached the limit on number of transmissions, transmit concurrently the received information with maximum transmit power. This continues until no more nodes are able to transmit due to the transmission limit. The result of this procedure is waves of the same information propagating back and forth through the network until the Glossy flood is terminated. This simple yet effective operation primitive is the reason why we try to make Glossy more energy efficient through cooperation and robust multicast beamforming while studying its impact on performance.

\section{Preliminaries}

Before starting our problem formulation, we explain the system model used throughout this work to build our optimization problem and coalition formation algorithm.

\subsection{System model}

Our approach can be viewed as an adaptive transmit diversity scheme. The transmission protocol starts, as in standard Glossy, with one node initiating the flood. This initial phase is a simple multicast transmission where one node transmits a message, and the nodes within a reception range receive it. After a successful reception of the message by, let us say, $K$ nodes, these nodes become transmitters in the next round. In standard Glossy, all $K$ nodes proceed to transmit the same information simultaneously in a non-cooperative way. Here, we propose to define $K_{1} \subseteq K$ of cooperating nodes which acquire CSI from the receiver through limited feedback, in order to perform multicast beamforming. The remaining $K_{2}=K-K_{1}$ nodes do not cooperate and operate in standard Glossy. Please note that $K_{1}=\emptyset$ is standard Glossy. An example of the system model is shown in Fig. 2, where $K=5$ nodes, which have successfully received and decoded the message from the last transmission, send the same data $x$ 


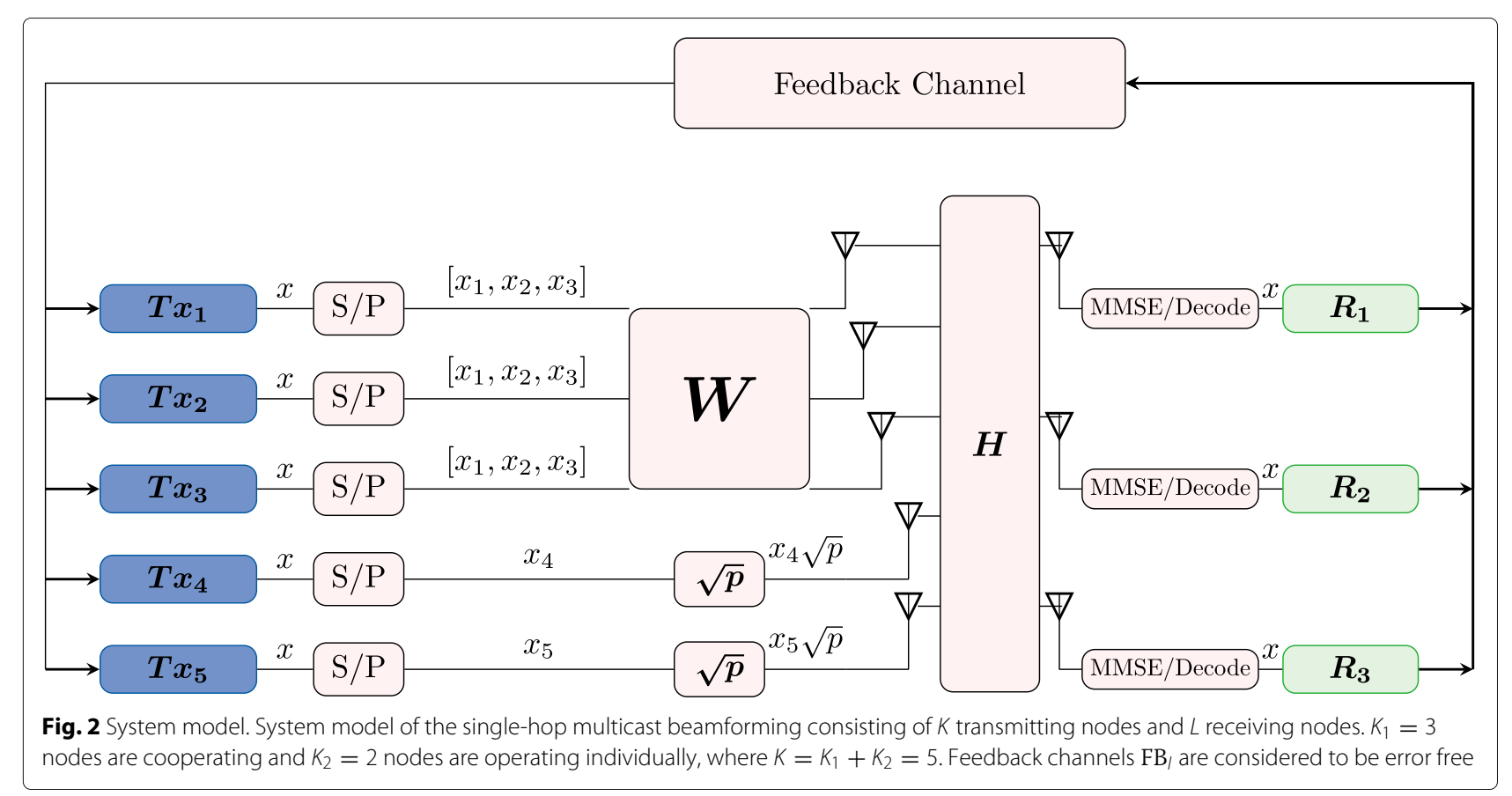

to $L=3$ receiving nodes. First, CSI is fed back from the receivers to the transmitters through a limited feedback channel as shown in Fig. 2 using random vector quantization as thoroughly explained in Section 2.2. Then, each transmitting node constructs an estimated channel matrix $\boldsymbol{H}$ using the acquired feedback and proceeds to calculate an energy-efficient coalition to which all or subset of the $K$ nodes ( $K_{1}$ nodes) can participate. The formation of this coalition is based on a greedy approach that can be executed at each transmitting node independently. This is explained in more details in Section 5. Afterwards, the information is divided into $K=5$ symbols where the first three symbols $\left[x_{1}, x_{2}, x_{3}\right]$ are cooperatively transmitted by $K_{1}$ nodes one to three, while symbols $x_{4}$ and $x_{5}$ are transmitted by $K_{2}$ nodes four and five, respectively. The coalition of the three nodes solves an energy efficiency optimization problem that will be formulated and discussed in Section 3 and cooperatively transmits the information forming a virtual antenna array that uses energy-efficient robust beamforming by applying the precoding matrix $W$, while the remaining two nodes operate in standard Glossy mode and transmit using maximum power $p$. The received signal at any receiver $l$ can be characterized as follows:

$$
y_{l}=\boldsymbol{x}^{T} \boldsymbol{U} \boldsymbol{h}_{l}+z_{l}
$$

where cooperation is controlled by the $K \times K$ matrix $\boldsymbol{U}=\operatorname{blockdiag}(\boldsymbol{W}, \sqrt{p} \boldsymbol{I}), \boldsymbol{x}^{T}=\left[x_{1} \ldots x_{5}\right]$ are the data symbols, $\boldsymbol{W}$ is the $K_{1} x K_{1}$ beamforming matrix, $\boldsymbol{h}_{l}^{T}=$ $\left[h_{1 l}, \ldots h_{5 l}\right]$ is the channel vector between receiver $l$ and the transmitting nodes, and $z_{l}$ is the receiver noise. The
[ $]^{T}$ describes the transpose operation. After the signal reception, interference cancelation methods (i.e., minimum mean square error (MMSE) and successive interference cancelation (SIC)) are applied at each receiving node before the original message $\boldsymbol{x}$ can be retrieved. The maximum rate achieved by applying such processing at any receiver node $l$ to the received signals can then be calculated as follows:

$R_{l}^{*}=\log \left(1+\frac{\left|\boldsymbol{x}^{T} \boldsymbol{U} \boldsymbol{h}_{l}\right|^{2}}{\sigma_{l}^{2}}\right)=\log \left(1+\frac{\boldsymbol{h}_{l}^{H} \boldsymbol{U}^{H} E\left[\boldsymbol{x}^{*} \boldsymbol{x}^{T}\right] \boldsymbol{U} \boldsymbol{h}_{l}}{\sigma_{l}^{2}}\right)$

where $E[]$ is the expectation operation, and $E\left[x^{*} x^{T}\right]$ is the transmit covariance matrix as shown in [28]. By applying MMSE and SIC techniques as demonstrated in ([29], ch. 10), it can be equivalently described as:

$$
R_{l}^{*}=\log \left(1+\frac{\left[\begin{array}{l}
h_{1 l} \\
h_{2 l} \\
h_{3 l}
\end{array}\right]^{H} W^{H} C_{3} W\left[\begin{array}{l}
h_{1 l} \\
h_{2 l} \\
h_{3 l}
\end{array}\right]}{\sigma_{l}^{2}}+\frac{\left|h_{l 4}\right|^{2} E\left[\left|x_{4}\right|^{2}\right]}{\sigma_{l}^{2}}+\frac{\left|h_{l 5}\right|^{2} E\left[\left|x_{5}\right|^{2}\right]}{\sigma_{l}^{2}}\right)
$$

where $h_{i l}$ is the channel coefficient from transmitter $i$ to receiver $l, C_{3}$ is the covariance matrix of data in the cooperating transmitters and is equal to the identity matrix $I$, the []$^{*}$ and []$^{H}$ describe the conjugate and conjugate transpose operations, respectively. Using the definition of Gram matrix ([30], p. 55), we can rewrite the achievable 
rate equation as follows:

$$
R_{l}^{*}=\log \left(1+\frac{\left[\begin{array}{l}
h_{1 l} \\
h_{2 l} \\
h_{3 l}
\end{array}\right]^{H} \boldsymbol{G}\left[\begin{array}{l}
h_{1 l} \\
h_{2 l} \\
h_{3 l}
\end{array}\right]}{\sigma_{l}^{2}}+\frac{\left|h_{l 4}\right|^{2} p}{\sigma_{l}^{2}}+\frac{\left|h_{l 5}\right|^{2} p}{\sigma_{l}^{2}}\right)
$$

where

$$
\boldsymbol{G}=\boldsymbol{W}^{H} \boldsymbol{W}
$$

is a positive semi-definite matrix. This can be generalized to the case of $K=K_{1}+K_{2}$ transmitters with $K_{1}$ cooperating nodes and $K_{2}$ non-cooperating nodes as follows:

$$
R_{l}^{*}=\log \left(1+\frac{\boldsymbol{h}_{l} \boldsymbol{G} \boldsymbol{h}_{l}^{H}}{\sigma_{l}^{2}}+\sum_{i=K_{1}+1}^{K} \frac{\left|h_{i l}\right|^{2} p}{\sigma_{l}^{2}}\right)
$$

where $\boldsymbol{h}_{l}^{T}=\left[h_{1}, h_{2} \ldots h_{K_{1}}\right], \boldsymbol{G}$ is a $K_{1} \times K_{1}$ positive semidefinite matrix, and $p$ is the maximum transmit power of the node. Moreover, for the case where all transmitting nodes are cooperating, the maximum achievable rate can be described as:

$$
R_{l}^{*}=\log \left(1+\frac{\boldsymbol{h}_{l} \boldsymbol{G} \boldsymbol{h}_{l}^{H}}{\sigma_{l}^{2}}\right)
$$

with $K \times K$ positive semi-definite matrix $\mathbf{G}$.

\subsubsection{Channel model}

In order to examine the effect of limited feedback on a complex communication scenario such as Glossy, we must specify our channel characteristics. We start with the signal received at receiver $l$, described in (1). Channel vectors of receivers 1 to $L$ with $\mathbf{h}_{l}^{T}=\left[h_{1 l}, h_{2 l}, \ldots . h_{K l}\right]$ and $h_{k l} \in \mathbb{C}$ is the channel gain from transmitter $k$ to receiver $l$ modeled as quasi-static, independent, and experience flatfading on the block length, which is a valid assumption for low-power wireless devices such as Zigbee ([31], E 5.3). These coefficients are calculated according to $h_{k l}=w_{k l} g_{k l}$, where small-scale fading $w_{k l}$ follows a complex normal distribution $\mathcal{C N}(0,1)$, and large-scale fading $g_{k l}$ depends on the distance $d_{k l}$ between transmitter $k$ and receiver $l$ ([31], E 5.3) as follows:

$$
g_{k l}= \begin{cases}40.2+20 \log \left(d_{k l}\right) & , d_{k l} \leq 8 \mathrm{~m} \\ 58.5+33 \log (d k l / 8) & , d_{k l}>8 \mathrm{~m}\end{cases}
$$

which corresponds to a path loss exponent of 2 for the first $8 \mathrm{~m}$ and a path loss exponent of 3.3 for distances larger than $8 \mathrm{~m}$. To ensure $g_{k l}>0$ according to (8), we consider only the topologies where the distance between any pair of nodes exceeds $0.1 \mathrm{~m}$, which is reasonable in real deployments [32]. We denote the normalized channel vector $\tilde{\mathbf{h}}_{l}=\frac{\mathbf{h}_{l}}{\left\|\mathbf{h}_{l}\right\|}$, where $\tilde{\mathbf{h}}_{l}$ is a unit vector that describes only the channel direction. Moreover, in our model, transmitters are assumed to know the channel quality information (CQI), represented by $\left\|\mathbf{h}_{l}\right\|$, perfectly, while each receiver $l$ is assumed to know local CSI $\mathbf{h}_{l}$ through pilot training $[33,34]$ at the beginning of transmission which is not the focus of this work. CSI is then quantized and shared with transmitting nodes through a limited feedback channel as described in Section 2.2. Finally, $z_{l}$ is the additive white Gaussian noise with a complex normal distribution $z_{l} \sim \mathcal{C N}\left(0, \sigma_{l}^{2}\right)$.

\subsection{CSI feedback}

Quantization is done using randomly generated vector quantization codebooks independently at each receiver. A codebook $\mathcal{C}$ contains $2^{B} K$-dimensional unit norm vectors randomly drawn from the isotropic distribution on the $K$-dimensional unit sphere where $\mathcal{C} \triangleq\left\{\mathbf{c}_{1}, \mathbf{c}_{2}, \ldots . \mathbf{c}_{2^{B}}\right\}$. In addition to being mathematically tractable, random vector quantization (RVQ) performs close to optimal quantization as feedback bits $B \rightarrow \infty$ [35]. This can be independently applied at each receiver $l$ where the closest vector $\mathbf{c}_{i}$ to the normalized real channel $\tilde{\mathbf{h}}_{l}$ is chosen. The index $i$ is then broadcasted to active transmitters using $B$ feedback bits. The RVQ process is performed such that the closest vector $\mathbf{c}_{i}$ to the normalized real channel $\tilde{\mathbf{h}}_{l}$ has to be chosen. We consider the Euclidean distance, defined as the norm of the difference between $\mathbf{c}_{i}$ and $\tilde{\mathbf{h}}_{l}$, as a measure of closeness. Each receiver must choose the closest vector $\mathbf{c}_{i}$ to the normalized channel $\tilde{\mathbf{h}}_{l}$ independently. This is done by solving the following problem:

$$
i_{l}=\arg \min _{1 \leq i \leq 2^{B}}\left\|\tilde{\mathbf{h}}_{l}-\mathbf{c}_{i}\right\|^{2}
$$

Since the norm of the error vector is:

$$
\left\|\delta_{l}\right\|^{2}=\left\|\tilde{\mathbf{h}}_{l}-\hat{\mathbf{h}}_{l}\right\|^{2}
$$

where the $\hat{\mathbf{h}}_{l}$ is the quantized channel vector based on the Euclidean distance, this manner of choosing the quantized channel vector results in the minimum error norm possible. Unfortunately, the statistical behavior of this error is not available in closed form.

\subsubsection{Feedback overhead}

Feedback is the operation of sharing quantized CSI between receivers and transmitters. Each receiver node $l$ must quantize and share its CSI using the feedback channel. Thanks to the broadcast nature of the wireless medium and assuming error-free feedback channels, it is safe to assume that any wireless signal received successfully by the furthest node, can be received by all other nodes, and the power needed for reliable transmission is at least: 


$$
P_{l}=\left(2^{B}-1\right) \max _{1 \leq j \leq K, j \neq l}\left|d_{l j}\right|^{2}
$$

where $d_{l j}$ is the distance from receiver $l$ to transmitter $j$, operator $|$.$| denotes the Euclidean norm, and P_{l}$ is defined as the transmission power needed to distribute CSI from receiver $l$ to all transmitters. The total feedback energy overhead is computed as follows:

$$
P_{\text {coop }}=\left(2^{B}-1\right) \sum_{l=1}^{L} \max _{1 \leq j \leq K, j \neq l}\left|d_{l j}\right|^{2}
$$

where we can see the exponential dependency of $P_{\text {coop }}$ on the number of feedback bits $B$ and linear dependency on the distances between receivers and transmitters. This means that increasing the number of feedback bits $B$ will result in a higher cooperation over head in terms of $P_{\text {coop }}$ but will also result in a more accurate estimation of the channels, leading to less transmit power. Therefore, there must be an optimum number of feedback bits $B$ that minimizes the total energy consumption and maximizes energy efficiency. This brings us to our problem statement, which we present in Section 2.3.

\subsection{Problem statement}

In order to efficiently manage the network resources and achieve high energy efficiency in the state-of-the-art Glossy [36, 37], we seek to analyze the feasibility of using power control and transmission cooperation schemes such as coherent multicast beamforming. To achieve our goal, we first look at the problem of limited feedback, where receiving nodes try to share their CSI with transmitting nodes using $B$ feedback bits. Increasing the number of feedback bits $B$ used in CSI sharing results in a better representation of the channels and lower quantization error. However, the consumed energy overhead by reliably transmitting these feedback bits increases as well. This results in a clear trade-off between the cooperation gain achieved by coherent multicast beamforming among the $K$ transmitting nodes and the consumed energy overhead due to CSI feedback. Therefore, there must exist an optimal $B$, dependent on network parameters such as topology, number of transmitters $K$, and density of the network, that maximizes energy efficiency. This allows us to solve a programming problem, that depends on the CSI uncertainty parameter $\epsilon_{l}$, and in turn optimal $B$, that maximizes energy efficiency under quality of service constraints (QoS) and guarantees successful reception as follows:

$$
\begin{aligned}
& \min _{\boldsymbol{G}} \operatorname{tr}(\boldsymbol{G}) \\
& \text { subject to }\left(\hat{\mathbf{h}}_{l}+\delta\right) \boldsymbol{G}\left(\hat{\mathbf{h}}_{l}+\delta\right)=\frac{\left(2^{R}-1\right) \sigma_{l}^{2}}{\left\|\mathbf{h}_{l}\right\|^{2}} ; \forall l \in \mathcal{L},
\end{aligned}
$$

$$
\forall \delta:\|\delta\| \leq \epsilon,
$$

$$
\boldsymbol{G} \succeq 0 .
$$

where all transmitters are assumed to be cooperating and minimizing the beamforming transmit powers $\operatorname{tr}(\boldsymbol{G})$ and the feedback power under three inequality constraints. Inequality (14) ensures the achievable rate robustness against CSI uncertainty, inequality (15) limits the maximum CSI uncertainty error, and inequality (16) insures that cooperation multicast beamforming covariance matrix $\boldsymbol{G}$ is positive semi-definite. This programming problem results in maximizing the energy efficiency and is solved and discussed in more details in Sections 3 and 4. Lastly, we look at the problem of choosing cooperating nodes. We investigate how the choice of cooperating transmitters $K_{1}$ affects energy efficiency, whether there exists an optimum coalition and how to form it.

\section{Transmit beamforming optimization under limited feedback}

In our previous work [5], we considered the case of multicast beamforming under the assumption of perfect CSI available at both transmitter and receiver. The following programming problem was solved for the optimal precoder $\mathbf{G}$ :

$$
\begin{array}{cl}
\min _{\boldsymbol{G}} & \operatorname{tr}(\boldsymbol{G}) \\
\text { subject to } & \mathbf{h}_{l} \boldsymbol{G} \mathbf{h}_{l}^{H} \geq\left(2^{R}-1\right) \sigma_{l}^{2} ; \forall l \in \mathcal{L} \\
& \boldsymbol{G} \geq 0
\end{array}
$$

where the inequality $\boldsymbol{G} \succeq 0$ means that $\boldsymbol{G}$ is a positive semi-definite matrix. The programming problem in (17) is a convex problem with a linear objective function, convex constraint set, and is formulated as explained in [9]. To solve it, we use CVX, a MATLAB package for solving convex programs $[38,39]$.

Unfortunately perfect CSI knowledge at the transmitter side is not always possible as in this work, where limited feedback is considered. Therefore, based on the available CSI provided to the transmitters through limited feedback and using the uncertainty model explained in Section 2.2, the transmitter has to estimate the real channels in order to calculate the appropriate beamformer. First, in order to satisfy the QoS constraints at each receiver $l$, the following must hold:

$$
\log \left(1+\frac{\mathbf{h}_{l} \mathbf{G h}_{l}^{H}}{\sigma_{l}^{2}}\right) \geq R ; \forall l \in \mathcal{L}
$$

The real channel $\mathbf{h}_{l}$ can be written in terms of the quantized channel direction $\hat{\mathbf{h}}_{l}$ and the channel norm $\left\|\mathbf{h}_{l}\right\|$ as such:

$$
\mathbf{h}_{l}=\left\|\mathbf{h}_{l}\right\| \tilde{\mathbf{h}}_{l}=\left\|\mathbf{h}_{l}\right\|\left(\hat{\mathbf{h}}_{l}+\delta_{l}\right)
$$

We assume that both $\hat{\mathbf{h}}_{l}$ and $\left\|\mathbf{h}_{l}\right\|$ are known by the transmitter. Therefore, after some algebraic manipulations, (18) can be written as: 


$$
\begin{aligned}
& \left(\hat{\mathbf{h}}_{l}+\delta_{l}\right) \boldsymbol{G}\left(\hat{\mathbf{h}}_{l}+\delta_{l}\right)^{H} \geq \frac{\left(2^{R}-1\right) \sigma_{l}^{2}}{\left\|\mathbf{h}_{l}\right\|^{2}} ; \\
& \forall l \in \mathcal{L}, \forall \delta_{l}:\left\|\delta_{l}\right\| \leq \epsilon_{l}
\end{aligned}
$$

where $\epsilon_{l}$ is an upper bound on the norm of quantization error. In order to design a beamformer that is robust against quantization error, the transmitters must minimize the transmit power while satisfying the rate constraints in (20). Using (20) and (17), the robust beamforming optimization problem can be formulated as in (13).

Since the quantization error vector $\delta_{l}$ is random and exact knowledge of its direction is not available at the transmitter, we modify the optimization problem mathematically so it can be solved numerically. To that purpose, we apply some simplification and algebraic modifications to the constraints in (20) similar to the analysis done in [40]. The robust beamforming optimization problem in (13) becomes equivalent to the following semi-definite programming problem which can be solved by a MATLAB tool for convex optimization known as CVX [38, 39]:

$$
\begin{array}{ll}
\min _{\mathbf{G Q}, \mu} & \operatorname{tr}(\boldsymbol{G}) \\
\text { subject to } & \hat{\mathbf{h}}_{l}(\boldsymbol{G}-\boldsymbol{Q}) \hat{\mathbf{h}}_{l}^{H}-\epsilon_{l}^{2} \mu \geq \frac{\left(2^{R}-1\right) \sigma_{l}^{2}}{\left\|\mathbf{h}_{l}\right\|^{2}} ; \\
& \forall l \in \mathcal{L}, \mu \geq 0, \boldsymbol{G} \geq 0
\end{array}
$$$$
\left[\begin{array}{lc}
\boldsymbol{Q} & \boldsymbol{G} \\
\boldsymbol{G} & \boldsymbol{G}+\mu \boldsymbol{I}
\end{array}\right] \geq 0
$$

Based on S-procedure and Schur complement as explained in Appendix A and B, respectively, we first transform the rate constraints in (20) as:

$$
\begin{aligned}
& \delta_{\mathbf{l}} \boldsymbol{G} \delta_{l}^{H}+2 \hat{\mathbf{h}}_{l} \boldsymbol{G} \delta_{l}^{H}+\hat{\mathbf{h}}_{l} \boldsymbol{G} \hat{\mathbf{h}}_{l}{ }^{H}-z \geq 0 ; \\
& \forall l \in \mathcal{L}, \forall \delta_{l}:-\left\|\delta_{l}\right\|^{2}+\epsilon_{l}^{2} \geq 0
\end{aligned}
$$

where $z=\frac{\left(2^{R}-1\right) \sigma_{l}^{2}}{\left\|\mathbf{h}_{l}\right\|^{2}}$, and $\|\delta\|^{2}=\delta^{H} \delta$. According to Sprocedure, this holds if and only if there exists $\mu \geq 0$ such that:

$$
\left[\begin{array}{cc}
\boldsymbol{G}+\mu \boldsymbol{I} & \boldsymbol{G} \tilde{\mathbf{h}}_{l} \\
\tilde{\mathbf{h}} \boldsymbol{G} & \tilde{\mathbf{h}}_{l} \boldsymbol{G} \tilde{\mathbf{h}}_{l}^{H}-\epsilon_{l}^{2} \mu-z
\end{array}\right] \succeq 0,
$$

This provides us with two separate cases for $\mu$. First, we examine the case where $\mu>0$. Using Schur complement, (23) can be written as:

$$
\begin{aligned}
& \tilde{\mathbf{h}}_{l} \boldsymbol{G} \tilde{\mathbf{h}}_{l}^{H}-\epsilon_{l}^{2} \mu-z-\tilde{\mathbf{h}}_{l} \boldsymbol{G}[\boldsymbol{G}+\mu \boldsymbol{I}]^{-1} \boldsymbol{G}_{\mathbf{h}_{l}}^{H} \geq 0 \\
& \tilde{\mathbf{h}}_{l} \boldsymbol{G} \tilde{\mathbf{h}}_{l}{ }^{H}-\tilde{\mathbf{h}}_{l} \boldsymbol{G}[\boldsymbol{G}+\mu \boldsymbol{I}]^{-1} \boldsymbol{G} \tilde{\mathbf{h}}_{l}^{H}-\epsilon_{l}^{2} \mu \geq z
\end{aligned}
$$

Second, we introduce $\boldsymbol{Q}$, where $\boldsymbol{Q} \succeq[\boldsymbol{G}+\mu \boldsymbol{I}]^{-1} \boldsymbol{G}$. The robust beamforming optimization problem in (13) can be rewritten as:

$$
\begin{array}{ll}
\min _{\boldsymbol{G Q}, \mu} & \operatorname{tr}(\boldsymbol{G}) \\
\text { subject to } & \hat{\mathbf{h}}_{l} \boldsymbol{G} \hat{\mathbf{h}}_{l}^{H}-\hat{\mathbf{h}}_{l} \boldsymbol{Q} \hat{\mathbf{h}}_{l}^{H}-\epsilon_{l}^{2} \mu \geq \frac{\left(2^{R}-1\right) \sigma_{l}^{2}}{\left\|\mathbf{h}_{l}\right\|^{2}} ; \\
& \forall l \in \mathcal{L}, \mu>0, \boldsymbol{G} \succeq 0 \\
& \mathbf{Q}-[\boldsymbol{G}+\mu \boldsymbol{I}]^{-1} \boldsymbol{G} \succeq 0
\end{array}
$$

By applying Schur complement to the constraint in (25), it can be transformed equivalently into:

$$
\begin{array}{ll}
\min _{\boldsymbol{G Q}, \mu} & \operatorname{tr}(\boldsymbol{G}) \\
\text { subject to } & \hat{\mathbf{h}}_{l}(\boldsymbol{G}-\boldsymbol{Q}) \hat{\mathbf{h}}_{l}^{H}-\epsilon_{l}^{2} \mu \geq \frac{\left(2^{R}-1\right) \sigma_{l}^{2}}{\left\|\mathbf{h}_{l}\right\|^{2}} ; \\
& \forall l \in \mathcal{L}, \mu>0, \boldsymbol{G} \geq 0 \\
& {\left[\begin{array}{l}
\boldsymbol{Q} \\
\boldsymbol{G} \mathbf{G}+\mu \boldsymbol{I}
\end{array}\right] \geq 0}
\end{array}
$$

Second, for $\mu=0$, (23) amounts to:

$$
\left[\begin{array}{cc}
\boldsymbol{G} & \boldsymbol{G} \tilde{\mathbf{h}}_{l} \\
\tilde{\mathbf{h}_{l}} \boldsymbol{G} & \tilde{\mathbf{h}}_{l} \boldsymbol{G} \tilde{\mathbf{h}}_{l}^{H}-z
\end{array}\right] \succeq 0
$$

and equivalently

$$
\left[\begin{array}{ll}
\tilde{\mathbf{h}}_{l}^{H} & 1
\end{array}\right]\left[\begin{array}{cc}
\boldsymbol{G} & \tilde{G}_{\tilde{\mathbf{h}}_{l}} \\
\tilde{\mathbf{h}_{l}} \boldsymbol{G} & \tilde{\mathbf{h}}_{l} \tilde{\mathbf{h}}_{l}^{H}-z
\end{array}\right]\left[\begin{array}{l}
-\tilde{\mathbf{h}}_{l} \\
1
\end{array}\right]=-z \geq 0
$$

which means that the constraints in (26) can be satisfied for the case of $\mu=0$ by having $Q=\boldsymbol{G}$ and can hereby be included into (26), arriving to the final result in (21). Algorithm 1 shows the operations done at each transmitting node after receiving the feedback signal.

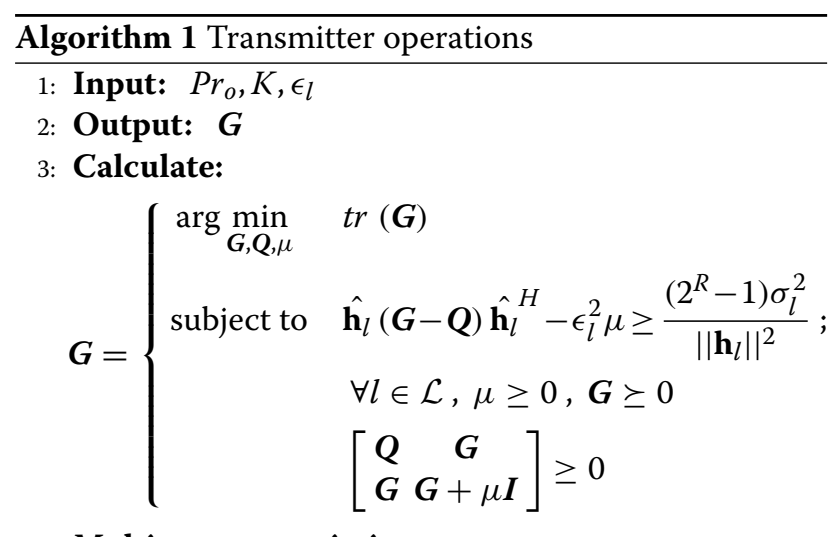




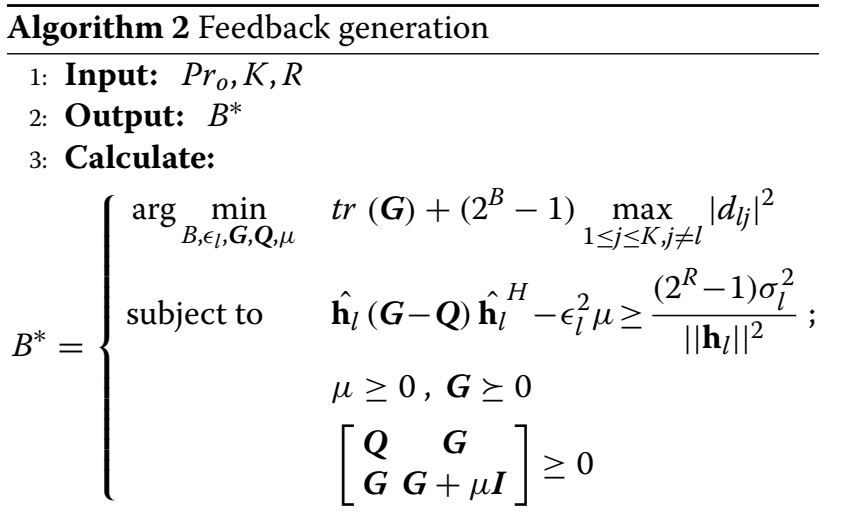

4: Transmit feedback with power $P_{l}=\left(2^{B^{*}}-\right.$ 1) $\max _{1 \leq j \leq K, j \neq l}\left|d_{l j}\right|^{2}$

\section{Maximizing energy efficiency through feedback optimization}

First, we evaluate the effect that the number of feedback bits $B$ has on energy efficiency of an arbitrary single hop within a Glossy flood. This is the first step towards optimizing the number of feedback bits $B$ from an energy efficiency point of view. To do so, we must define our performance measures. So, we begin by defining energy efficiency as introduced in [41] as follows:

$$
E E=\frac{\text { Goodput }}{\text { Total energy consumption }}
$$

where, in our scenario, the average rate is defined, similar to the formulation in [42], as the average successfully received rate at each receiver, and the total energy consumption is the sum of transmit energies for all $K$ transmitters and the cooperation overhead $P_{\text {coop }}$ as defined in (12). The energy efficiency can be rewritten as such:

$$
E E=\frac{\left(1-\operatorname{Pr}_{o}\right) R}{\operatorname{tr}(\boldsymbol{G})+P_{\text {coop }}}
$$

where $\operatorname{Pr}_{o}$ is the outage probability fixed to an application specific minimum acceptable value, and $R$ is the source rate fixed at $250 \mathrm{kbps}$ as in the IEEE 802.15.4 standard [31].

In order to maximize energy efficiency, we need to consider the following optimization problem:

$$
\max _{B(\epsilon)} E E=\frac{\left(1-\operatorname{Pr}_{o}\right) R}{\operatorname{tr}(\boldsymbol{G})+P_{\text {coop }}}
$$

Due to the fixed nominator, this problem is equivalent to:

$$
\min _{B(\epsilon)} \operatorname{tr}(\boldsymbol{G})+\left(2^{B(\epsilon)}-1\right) \max _{1 \leq j \leq K, j \neq l}\left|d_{l j}\right|^{2}
$$

Unfortunately, due to the lack of a closed form expression for $B$ in terms of $\epsilon$, this optimization problem can not be simultaneously solved for $\boldsymbol{G}$ and $B$. Therefore, an offline database created using network statistics is saved at each node, where $\epsilon$ can be chosen as a function of $K, B$, and $P r_{o}$. An example of the offline database is shown in Fig. 3 for $P r_{o}=5 \%$. After that, the programming problem in (32) can be solved independently at each receiver node for each $B$ in order to find $B^{*}$ that achieves the minimum consumed energy for the application specific $P r_{o}$ requirement. The feedback generation algorithm in Algorithm 2 shows the operational steps carried out at each receiver node, while the flow chart in Fig. 4 shows the protocol operations flow at each node.

A simple example can be seen in Fig. 5 with four transmitting nodes waiting for receiver feedback and three receiving nodes executing Algorithm 2. Due to different positions, each receiver will have a relation between consumed energy and $B$. The dependency of consumed energy on the number of feedback bits $B$ for each receiver is calculated and shown in Fig. 6 . We can see there that it is possible that $B^{*}$ would be different for each receiver depending on its position in the network. In our example, receivers 1,2 , and 3 will choose 2, 3, and 5 bits, respectively, for feedback transmission.

Error Database Example. An example of the offline database saved at each node where $\epsilon$ can be chosen as a function of $K, B$, and $P r_{o}$. In this example, $P r_{o}$ is set to $5 \%$

\section{Coalition formation}

The proposed enhancements of the Glossy approach are divided into two parts. The first part is a feedback generation at receiving nodes, followed by multicast beamforming at transmitting nodes as explained in Sections 3 and 4 , respectively. The second part is a coalition formation strategy that chooses cooperating transmitters in order to maximize energy efficiency. In this section, we analyze the effect of coalition size and participant transmitters on energy efficiency and if there exists an optimal choice of cooperating nodes that maximizes energy efficiency.

For this part, we allow a subset of all transmitting nodes to cooperate. Our goal is to show the possibility that a certain cooperating group of transmitters (coalition) exists to maximize energy efficiency. It is important to note that this study is to show the effectiveness of coalition formation and not to demonstrate how it can be implemented in a distributed fashion. First, feedback operation is performed as explained in Section 2.2, where the receivers provide the CSI to the $K$ transmitters. In order to minimize the energy consumed in the cooperation phase, the transmitter furthest away from all receivers is removed from the coalition. If this elimination operation improves the energy efficiency of the transmission, the next furthest away transmitter is removed. Elimination stops when the energy efficiency starts to deteriorate and the coalition is 


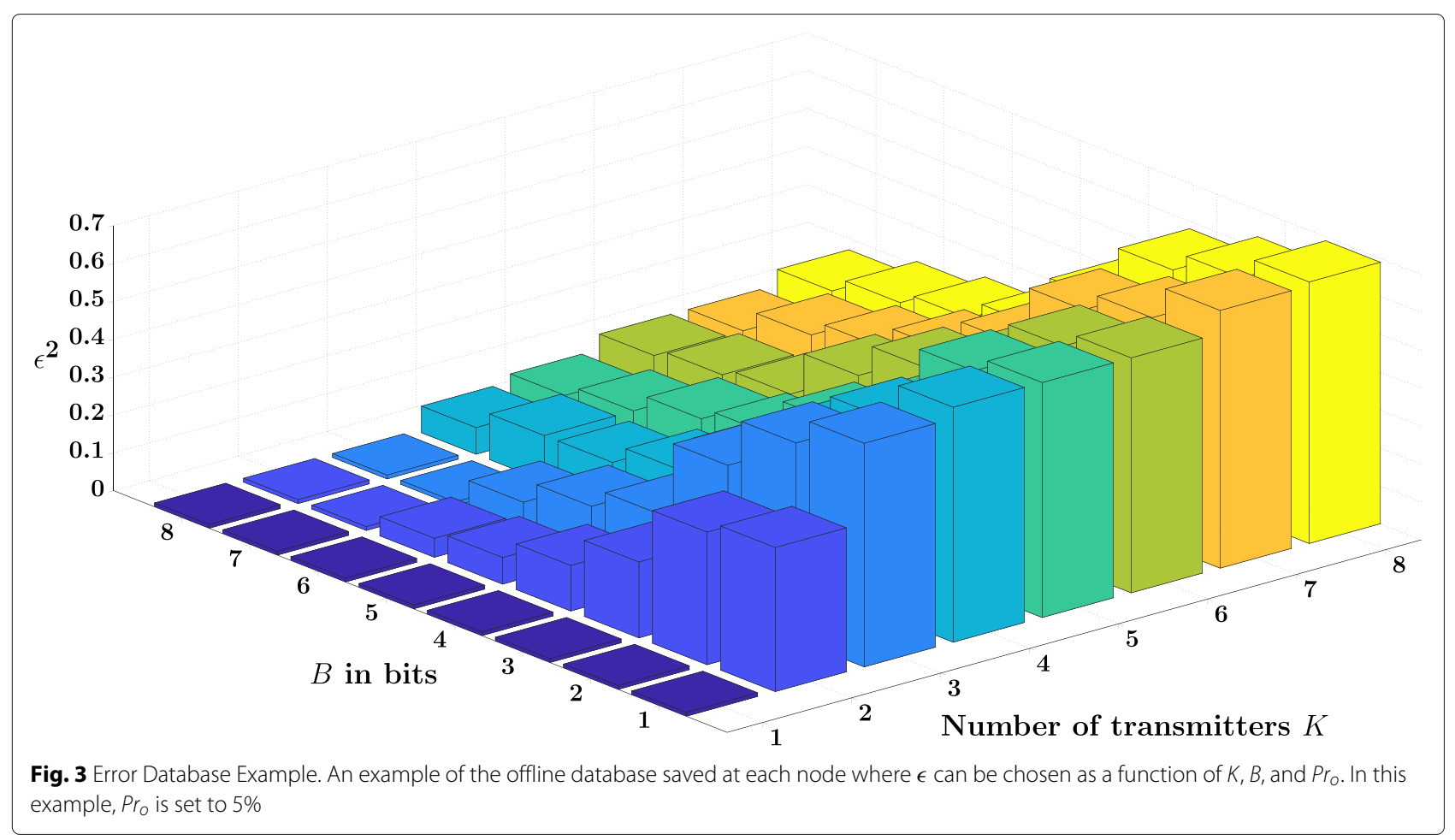

returned to the last step. This greedy transmitter selection operation is explained in Algorithm 3.

This operation results in a coalition of $K$ or less transmitters. The effect of this enhancement on energy efficiency and energy consumption compared to both normal Glossy and Glossy with transmit beamforming will be demonstrated in Section 6.

\section{Results and discussion}

We evaluate the performance of the proposed approach in two different cases. First, we consider an intermediate

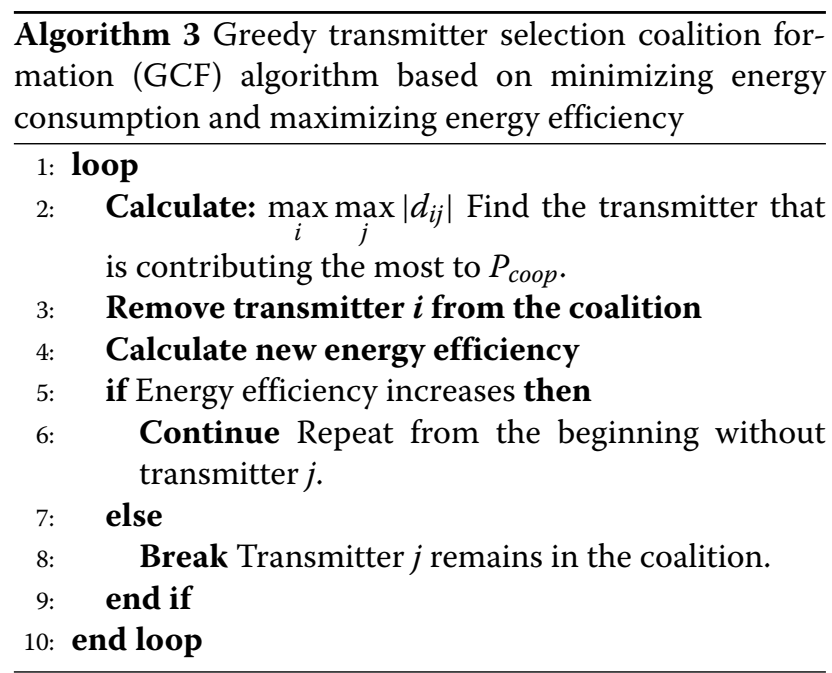

stage in a Glossy flood with $K$ transmitters and $L$ receivers. This case is considered for Fig. 7. Second, we consider a complete Glossy flood in a network of $\lambda$ nodes, where a random node initiates the flood. Then, we apply the standard Glossy and the robust multicast beamforming approach with and without coalition formation and evaluate the performance of each approach at the end of the flood, as in the case of Figs. 8 and 9.

We generate network topologies using a binomial point process with $\lambda=k+L$ nodes randomly and independently deployed in a square area. We then evaluate energy efficiency using robust multicast beamforming for different values of network side length in meters. Our results are averaged over 100 independent realizations of the network for the same $\lambda$. Irrespective of the topology, all transmitting nodes have a source rate $R=250 \mathrm{kbps}$ which corresponds to the default setting as prescribed by the IEEE 802.15.4 standard. The first step to optimize the system is solving the programming problem in (21) for each receiver independently. After that, each receiver chooses the number of feedback bits $B$ that minimizes energy consumption. This is shown together with the algorithm steps in Section 3.

\subsection{Tightness of bounds}

The programming problem in (21) is designed to minimize transmit power under QoS constraints. However, minimizing transmit power does not always mean an increase in energy efficiency. Other factors affect energy 


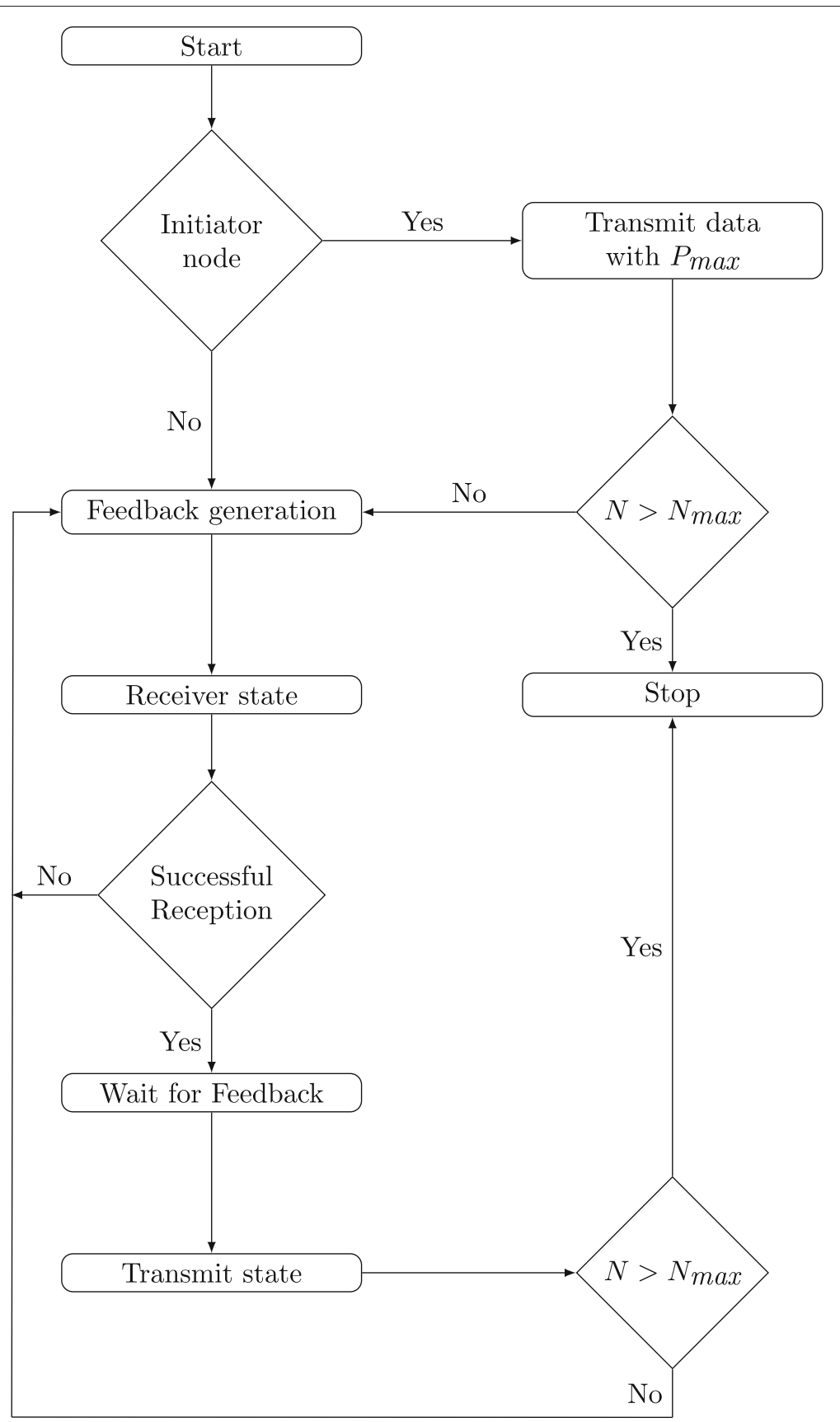

Fig. 4 Protocol operation. Commands executed at any node during the proposed protocol

efficiency such as outage probability and feedback overhead $P_{\text {coop }}$ which can be seen in (30). Modifying the optimization problem depends on the reliability requirements of the application. For example, in critical applications, an upper bound on the outage is needed. Therefore, we use the CDF of $\|\delta\|^{2}$ acquired by empirical means where outage probability is upper bounded as such $\operatorname{Pr}_{o} \leq$ $\operatorname{Pr}\left(\|\delta\|^{2}>\epsilon^{2}\right)$ and we solve for $\epsilon(B, K)$. This bound is not a tight bound because of the random direction of the error $\delta$, which can result in a better or worse quantized channel $\tilde{\mathbf{h}}_{l}$ than the real channel $\mathbf{h}_{l}$, depending on the direction of the error. Using this approach of accounting for worst case performance results sometimes in over satisfying the reliability requirements, hence a loss in terms of energy efficiency. However, the system can be solved for different number of feedback bits $B$ and ultimately find the optimum $B^{*}$ that maximizes energy efficiency while maintaining a maximum outage probability $\operatorname{Pr}_{o}$. The 
tightness of our bound can be seen in Fig. 7, where the reception ratio resulting from solving our robust optimization problem in (21) is calculated for different values of $\epsilon^{2}$. This is compared in the same plot with the empirical CDF of $\|\delta\|^{2}$ for the simulation scenario of $K=3$ and $B=4$.

It is critical here to emphasize the relationship between the CDF of $\epsilon^{2}$ and the reception ratio. Assuming that we use an $\epsilon^{2}$ corresponding to a $90 \%$ in the CDF, this means that $90 \%$ of the square of the quantization error norm is indeed below this value, in turn, resulting in a successful reception $90 \%$ of the time. However, It is easy to notice that achieving 90\% reception ratio requires a smaller $\epsilon^{2}$ than the one retrieved from the CDF information available at the transmitter. This bound can be tightened further by means of iterated optimization, where instead of minimizing $\|\delta\|^{2}$, we minimize $\delta^{H} \boldsymbol{G} \delta$. This results in recursively choosing a quantized channel $\tilde{\mathbf{h}}_{l}$ and a beamformer $\boldsymbol{G}$ that further maximize energy efficiency under rate constraints. We leave this approach for future works, and we focus on optimizing the number of feedback bits $B$ using offline databases stored at each node as explained in Section 3.

\subsection{System level performance}

We now test the performance of our proposed algorithm against state-of-the art Glossy. We consider nine sensor nodes randomly deployed in a square network with varying side length. One randomly chosen node initiates a flood with a required minimum reception ratio of $95 \%$ $\left(P r_{o}=5 \%\right)$, then energy consumption and energy efficiency are calculated at the end of the flood for both Glossy and the proposed algorithm. Both metrics are plotted in Figs. 8 and 9 against network side length in meters. Since the number of deployed nodes is fixed, varying the network side length can be seen as varying the network density. First, we notice an increase in power consumption and decrease in energy efficiency for less dense scenarios. This is a result of the flood generating more waves to reach the edges of the network. For example, in a very dense network, once a node initiates a flood, it is very probable that all nodes in the network will receive the information, hence the flood ends in two waves. However, for less dense scenarios, less nodes will receive the data successfully from the initiating node, resulting in more waves and consuming more energy. Second, energy efficiency behavior in Fig. 8 shows that our proposed algorithm greatly outperforms Glossy in dense networks, where nodes are close to each other and the cost of feedback (12) is not too high. This is expected since the feedback power needed for cooperation depends on the distance between nodes. On the other hand, in less dense scenarios, our proposed algorithm faces a degradation in performance due to the increase in feedback energy consumption. This shows that the proposed algorithm is best suited for dense networks scenarios where cooperation provides an edge in energy efficiency.

\section{Conclusions}

We have studied the problem of robust multicast beamforming in Glossy under imperfect CSI and limited feedback. It was shown that coherent multicast beamforming can lead to significant gains in terms of energy efficiency. By studying the effect of number of feedback bits $B$ on the performance, we show that there exists an optimal $B$ that maximizes energy efficiency. Moreover, the problem was solved for a specific maximum allowed outage probability. The results show that stricter outage requirements, result

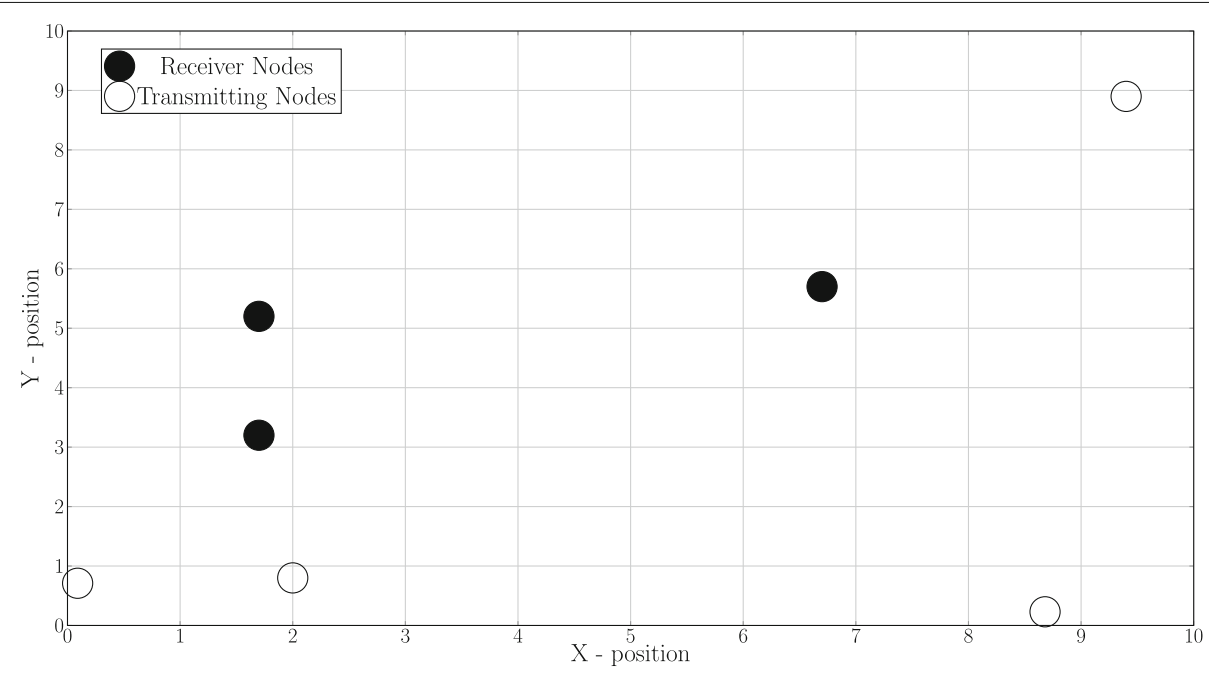

Fig. 5 Network example. Sensor network example with four transmitters in white and three receivers in black. Receivers generate feedback to provide transmitters with imperfect CSI 


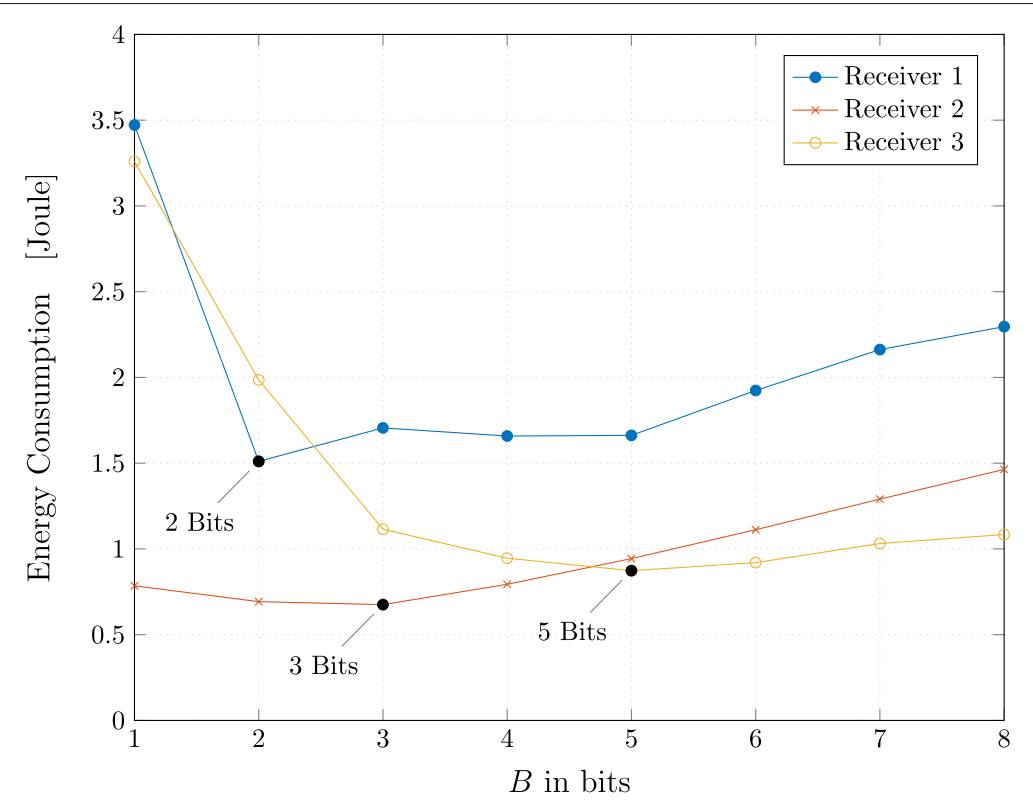

Fig. 6 Energy consumption. Energy consumption of feedback and multicast transmission of four transmitters calculated at three different receivers for different number of quantization bits $B$. Each receiver chooses $B^{*}$ that minimizes energy consumption

in higher values of optimal $B^{*}$. The upper bound used on the outage probability can be tightened using iterative beamforming, resulting in lower transmit power while still satisfying the rate constraints. However, we leave this for future investigations. Finally, a study was performed to examine the effect of selecting certain transmitting nodes for cooperation on energy efficiency. The simulation results showed that energy efficiency can be further improved by reducing the number of cooperating transmitters by means of greedy transmitter selection. The exact distributed implementation of mentioned algorithm is left for future efforts.

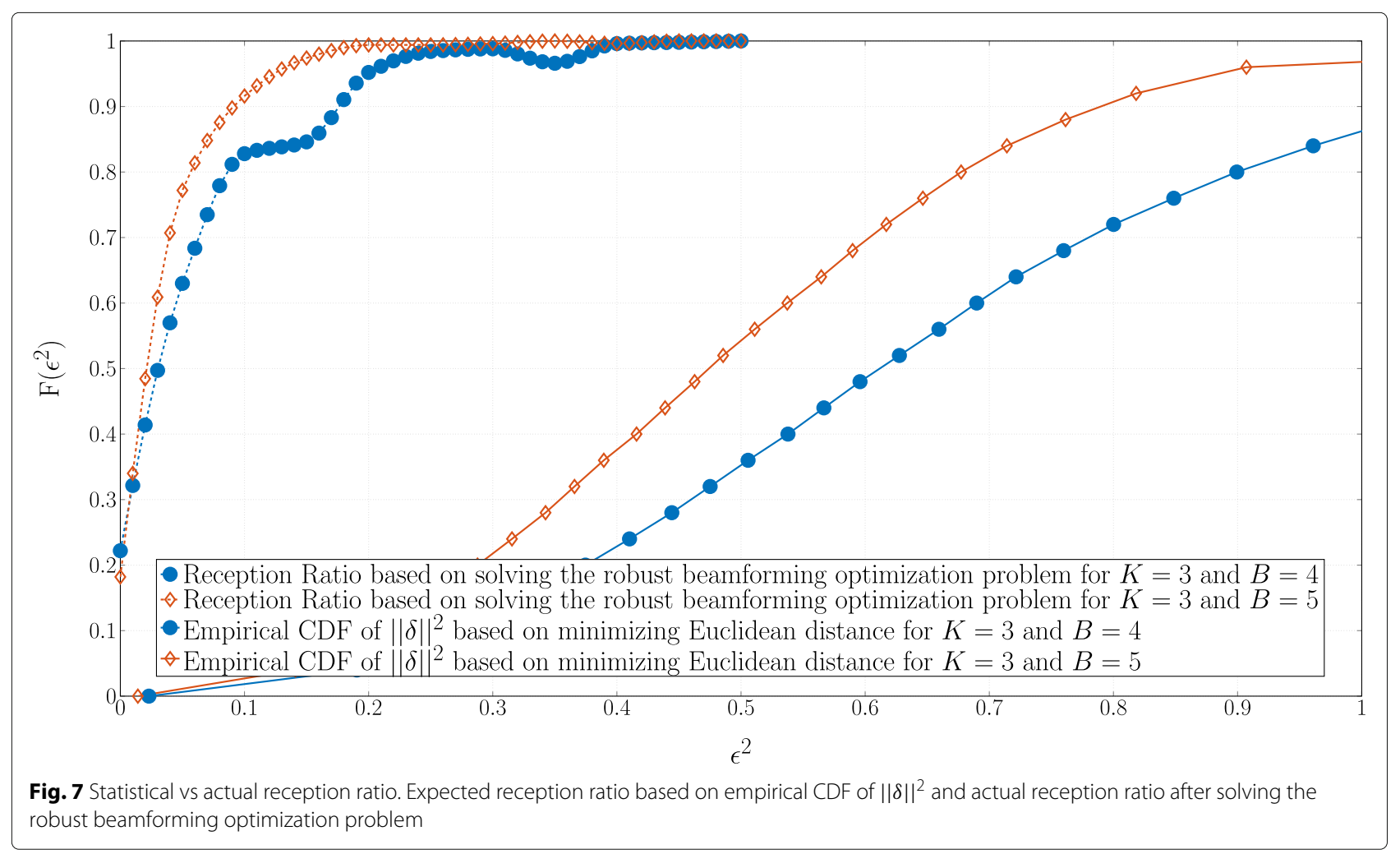




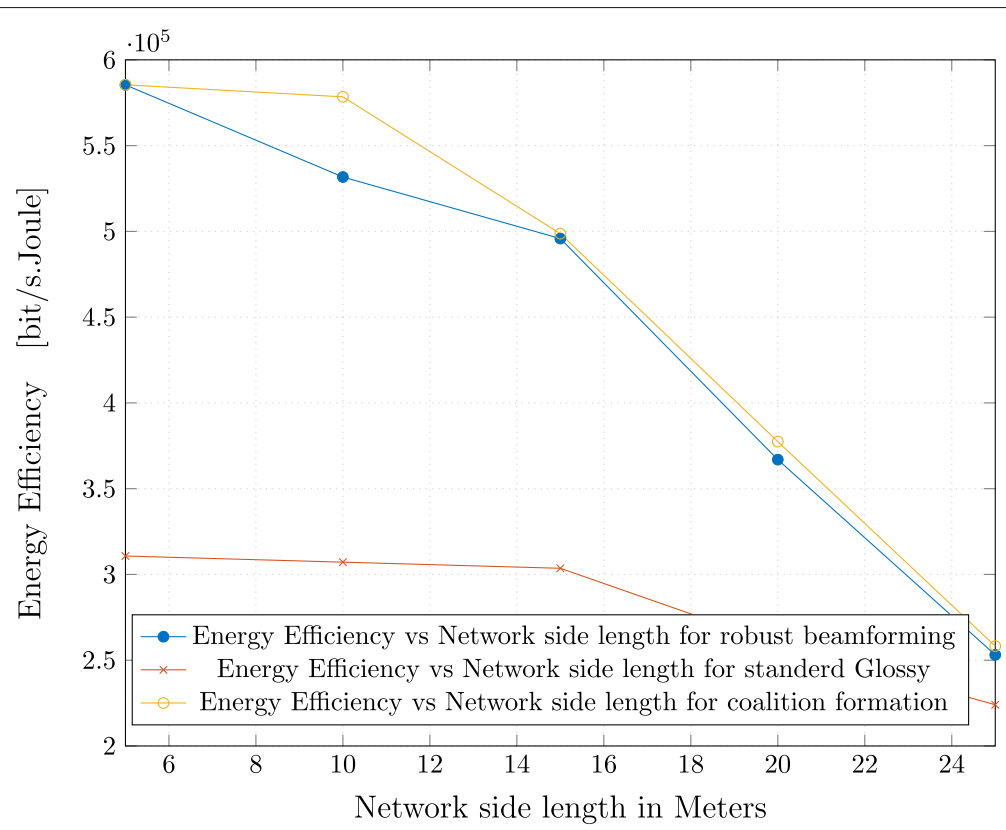

Fig. 8 Energy efficiency. Energy efficiency in bit/s. Joule evaluated at different network side lengths for standard Glossy, robust beamforming, and robust beamforming with coalition formation

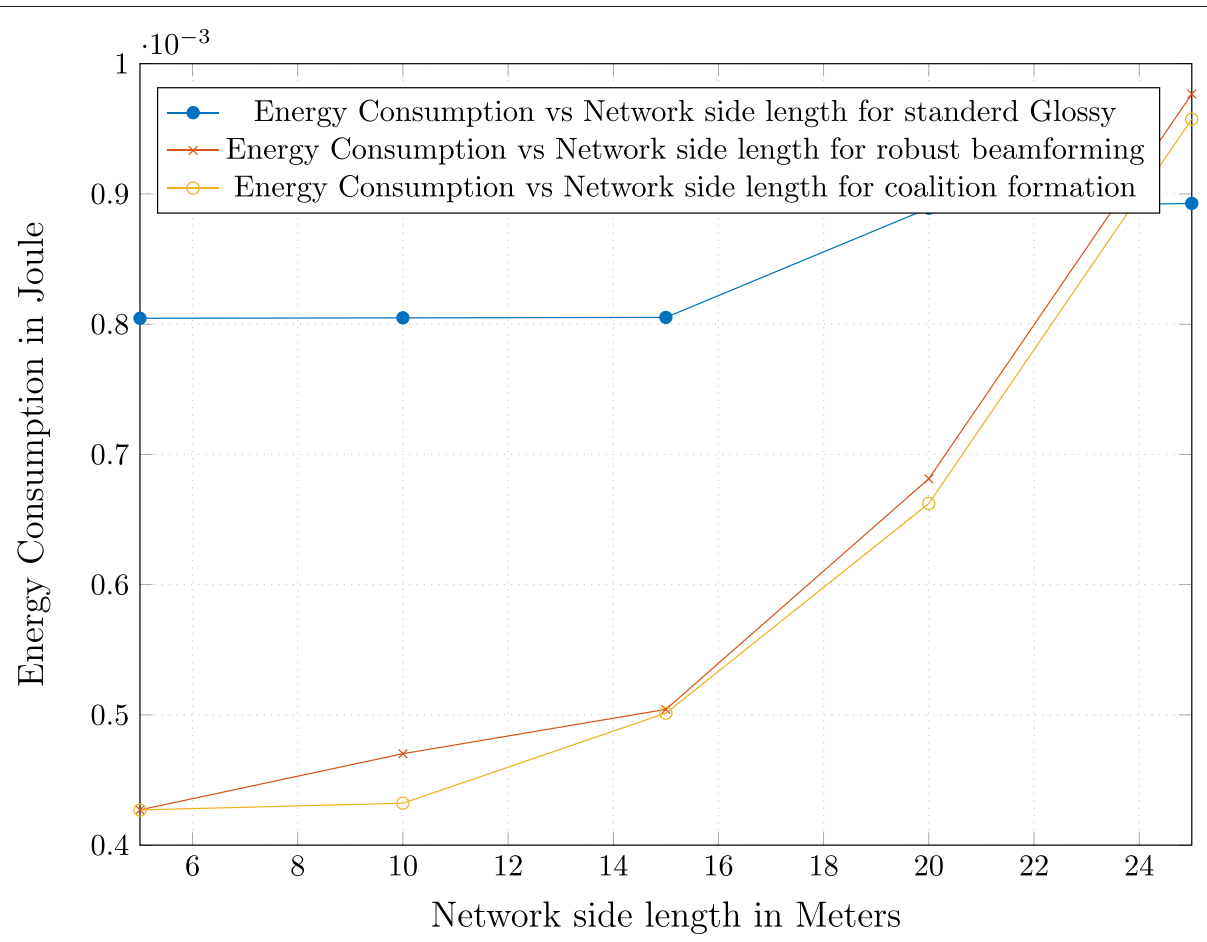

Fig. 9 Energy consumption. Energy consumption in Joule evaluated at different network side lengths for standard Glossy, robust beamforming, and robust beamforming with coalition formation 


\section{Appendix 1: S-procedure}

The S-procedure gives conditions under which a particular quadratic inequality is a consequence of another quadratic inequality [43]. Let $\boldsymbol{A}_{\mathbf{1}}$ and $\boldsymbol{A}_{\mathbf{2}}$ be symmetric matrices, $\mathbf{b}_{\mathbf{1}}$ and $\mathbf{b}_{\mathbf{2}}$ be vectors, and $c_{1}$ and $c_{2}$ be constants. Assuming there exists some $\mathbf{x}$ such that:

$$
\mathbf{x}^{H} \boldsymbol{A}_{\mathbf{1}} \mathbf{x}+2 \mathbf{b}_{\mathbf{1}} \mathbf{x}+c_{1} \geq 0
$$

then the following holds:

$$
\mathbf{x}^{H} \boldsymbol{A}_{2} \mathbf{x}+2 \mathbf{b}_{2} \mathbf{x}+c_{2} \geq 0
$$

if and only if there exists a $\mu \geq 0$, such that:

$$
\left[\begin{array}{ll}
\boldsymbol{A}_{2} & \mathbf{b}_{2} \\
\mathbf{b}_{2}{ }^{H} & c_{2}
\end{array}\right]-\mu\left[\begin{array}{ll}
\boldsymbol{A}_{1} & \mathbf{b}_{1} \\
\mathbf{b}_{1}{ }^{H} & c_{1}
\end{array}\right] \succeq 0
$$

\section{Appendix 2: Schur complement}

The Schur complement is defined as follows. Assume having:

$$
M=\left[\begin{array}{ll}
A & B \\
C & D
\end{array}\right] \succeq 0
$$

where $A, B, C$, and $D$ are matrices, and $D$ is invertible. Then, the Schur complement of $\boldsymbol{D}$ in $\boldsymbol{M}$ is:

$$
\boldsymbol{A}-\boldsymbol{B} \boldsymbol{D}^{-1} \boldsymbol{C} \succeq 0
$$

However, if $\boldsymbol{A}$ is invertible, then the Schur complement of $A$ in $M$ is:

$$
D-C A^{-1} B \succeq 0
$$

\section{Abbreviations}

CSI: Channel state information; CQI: Channel quality information; DCBF: Distributed collaborative beamforming; D2D: Device-to-device; FOS: Flooding overlay structure; GCF: Greedy coalition formation; loT: Internet of Things; LR-WPANs: Low-rate wireless personal access networks; MMSE: Minimum mean square error; MTC: Machine-type communication; QoS: Quality of service; SIC: Successive interference cancelation

\section{Acknowledgements}

The work of E. Jorswieck was supported partly by the German Research Foundation, Deutsche Forschungsgemeinschaft, Collaborative Research Center 912, through the Highly Adaptive Energy-Efficient Computing.

\section{Funding}

This work was supported by the German Research Foundation (DFG) within the Cluster of Excellence "Center for Advancing Electronics Dresden (cfaed)", through Priority Programs 1914, and by the European Union's Horizon 2020 research and innovation program under the Marie Sklodowska-Curie project 641985 (ETN-5Gwireless).

\section{Availability of data and materials}

The paper is self-contained, since we provide the protocol operations that can be used to reproduce the results presented with the help of Section 6 where numerical results and parameter settings are described in details.

\section{Authors' contributions}

AA derived the equations, constructed the algorithms, and performed the system simulations. EJ revised the equations and supervised and reviewed the paper. Both authors participated in this work and approved the final manuscript.

\section{Competing interests}

The authors declare that they have no competing interests.

\section{Publisher's Note}

Springer Nature remains neutral with regard to jurisdictional claims in published maps and institutional affiliations.

Received: 21 February 2019 Accepted: 16 April 2019

Published online: 11 June 2019

\section{References}

1. H. Yetgin, K. T. K. Cheung, M. El-Hajjar, L. H. Hanzo, A survey of network lifetime maximization techniques in wireless sensor networks. IEEE Commun. Surv. Tutor. 19(2), 828-854 (2017). https://doi.org/10.1109/ COMST.2017.2650979

2. A. B. Noel, A. Abdaoui, T. Elfouly, M. H. Ahmed, A. Badawy, M. S. Shehata, Structural health monitoring using wireless sensor networks: a comprehensive survey. IEEE Commun. Surv. Tutor. 19(3), 1403-1423 (2017). https://doi.org/10.1109/COMST.2017.2691551

3. S. Buzzi, C. L. I, T. E. Klein, H. V. Poor, C. Yang, A. Zappone, A survey of energy-efficient techniques for $5 \mathrm{G}$ networks and challenges ahead. IEEE J. Sel. Areas Commun. 34(4), 697-709 (2016). https://doi.org/10.1109/JSAC. 2016.2550338

4. M. Simsek, A. Aijaz, M. Dohler, J. Sachs, G. Fettweis, 5G-enabled tactile internet. IEEE J. Sel. Areas Commun. 34(3), 460-473 (2016). https://doi. org/10.1109/JSAC.2016.2525398

5. A. Abdelkader, E. Jorswieck, M. Zimmerling, in European Wireless 2017; 23th European Wireless Conference. Centralized and distributed optimum power control and beam-forming in network flooding, (2017), pp. 1-6

6. F. Ferrari, M. Zimmerling, L. Thiele, O. Saukh, in Proc. of the ACM/IEEE IPSN Efficient network flooding and time synchronization with Glossy, (2011)

7. E. Koyuncu, C. Remling, X. Liu, H. Jafarkhani, Outage-optimized multicast beamforming with distributed limited feedback. IEEE Trans. Wirel. Commun. 16(4), 2069-2082 (2017). https://doi.org/10.1109/TWC.2016. 2629485

8. R. F. Schaefer, H. Boche, Physical layer service integration in wireless networks : signal processing challenges. IEEE Signal Proc. Mag. 31(3), 147-156 (2014). https://doi.org/10.1109/MSP.2013.2271190

9. N. D. Sidiropoulos, T. N. Davidson, Z.-Q. Luo, Transmit beamforming for physical-layer multicasting. IEEE Trans. Signal Proc. 54(6), 2239-2251 (2006). https://doi.org/10.1109/TSP.2006.872578

10. A. B. Gershman, N. D. Sidiropoulos, S. Shahbazpanahi, M. Bengtsson, B. Ottersten, Convex optimization-based beamforming. IEEE Signal Proc Mag. 27(3), 62-75 (2010). https://doi.org/10.1109/MSP.2010.936015

11. J.Zander, Performance of optimum transmitter power control in cellular radio systems. IEEE Trans. Veh. Technol. 41(1), 57-62 (1992). https://doi. org/10.1109/25.120145

12. S. Stanczak, M. Wiczanowski, H. Boche, Fundamentals of resource allocation in wireless networks: theory and Algorithms, 2nd edn. (Springer Publishing Company, Incorporated, New York City, 2009)

13. R. Mudumbai, D. R. B. lii, U. Madhow, H. V. Poor, Distributed transmit beamforming: challenges and recent progress. IEEE Commun. Mag. 47(2), 102-110 (2009). https://doi.org/10.1109/MCOM.2009.4785387

14. J. Valenzuela-Valdés, F. Luna, R. M. Luque-Baena, P. Padilla, in 2014 IEEE 3rd International Conference on Cloud Networking (CloudNet). Saving energy in WSNs with beamforming, (2014), pp. 255-260. https://doi.org/10.1109/ CloudNet.2014.6969001

15. H. Ochiai, P. Mitran, H. V. Poor, V. Tarokh, Collaborative beamforming for distributed wireless ad hoc sensor networks. IEEE Trans. Signal Proc. 53(11), 4110-4124 (2005). https://doi.org/10.1109/TSP.2005.857028

16. H. Ochiai, P. Mitran, H. V. Poor, V. Tarokh, in Information Theory Workshop. Collaborative beamforming in ad hoc networks, (2004), pp. 396-401. https://doi.org/10.1109/ITW.2004.1405336

17. G. Barriac, R. Mudumbai, U. Madhow, in Third International Symposium on Information Processing in Sensor Networks, 2004. IPSN 2004. Distributed beamforming for information transfer in sensor networks, (2004), pp. 81-88. https://doi.org/10.1109//PSN.2004.238786

18. X. Zhang, K. G. Shin, Cooperation without synchronization: practical cooperative relaying for wireless networks. IEEE Trans. Mob. Comput. 14(5), 937-950 (2015). https://doi.org/10.1109/TMC.2014.2341611

19. B. B. Haro, S. Zazo, D. P. Palomar, Energy efficient collaborative beamforming in wireless sensor networks. IEEE Trans. Signal Proc. 62(2), 496-510 (2014). https://doi.org/10.1109/TSP.2013.2288080

20. S. Schedler, V. Kuehn, in 201411 th International Symposium on Wireless Communications Systems (ISWCS). Resource allocation for distributed 
beamforming with multiple relays and individual power constraints, (2014), pp. 1-5. https://doi.org/10.1109/ISWCS.2014.6933309

21. J. Ding, E. Dutkiewicz, X. Huang, G. Fang, in 2015 IEEE 81st Vehicular Technology Conference (VTC Spring). Energy-efficient distributed beamforming in uwb based implant body area networks, (2015), pp. 1-5. https://doi.org/10.1109/NTCSpring.2015.7145813

22. P. Ubaidulla, A. Chockalingam, in 2009 IEEE 20th International Symposium on Personal, Indoor and Mobile Radio Communications. Robust distributed beamforming for wireless relay networks, (2009), pp. 2345-2349. https:// doi.org/10.1109/PIMRC.2009.5449974

23. L. Wang, C. Zhang, J. Zhang, G. Wei, in Proceedings of the 5th International Conference on Wireless Communications, Networking and Mobile Computing, WiCOM'09. Distributed beamforming with limited feedback in regenerative cooperative networks (IEEE Press, Piscataway, 2009), pp. 1301-1304. http://dl.acm.org/citation.cfm?id=1736862.1737177

24. Y.-C. Tseng, S.-Y. Ni, Y.-S. Chen, J.-P. Sheu, The broadcast storm problem in a mobile ad hoc network. Wirel. Netw. 8(2), 153-167 (2002). https://doi. org/10.1023/A:1013763825347

25. S. Ahn, Y. Lim, H. Yu, in 2008 International Conference on Information Networking. Energy-efficient flooding mechanisms for the wireless sensor networks, (2008), pp. 1-5. https://doi.org/10.1109/ICOIN.2008.4472783

26. P. Levis, N. Patel, D. Culler, S. Shenker, in In Proceedings of the First USENIX/ACM Symposium on Networked Systems Design and Implementation (NSDI. Trickle: A self-regulating algorithm for code propagation and maintenance in wireless sensor networks, (2004), pp. 15-28

27. A. Abdelkader, J. Richter, E. A. Jorswieck, M. Zimmerling, in Proceedings of the International Conference on Computer Communications and Networks. Multi-flow glossy: physical-layer network coding meets embedded wireless systems, (Vancouver, 2017)

28. E. A. Jorswieck, H. Boche, Optimal transmission with imperfect channel state information at the transmit antenna array. Wirel. Pers. Commun. 27(1), 33-56 (2003). https://doi.org/10.1023/A:1026048118953

29. D. Tse, P. Viswanath, Fundamentals of wireless communication. (Cambridge University Press, New York, 2005)

30. D. G. Luenberger, Optimization by Vector Space Methods, 1st edn. (Wiley, New York, 1997)

31. IEEE standard for information technology- local and metropolitan area networks- specific requirements- part 15.4: Wireless I (MAC) and (PHY) specifications for low rate (WPANs). IEEE Std 802.15.4-2006

32. M. Ceriotti, et al, in ACM/IEEE IPSN. Monitoring heritage buildings with wireless sensor networks: the Torre Aquila deployment, (2009)

33. K. Y. Wang, T. H. Chang, W. K. Ma, C. Y. Chi, in 2012 IEEE International Conference on Acoustics, Speech and Signal Processing (ICASSP). Optimal transmission strategy for outage rate maximization in MISO fading channels with training, (2012), pp. 2945-2948. https://doi.org/10.1109/ ICASSP. 2012.6288533

34. A. Alkhateeb, O. E. Ayach, G. Leus, R. W. Heath, Channel estimation and hybrid precoding for millimeter wave cellular systems. IEEE J. Sel. Top. Signal Proc. 8(5), 831-846 (2014). https://doi.org/10.1109/JSTSP.2014. 2334278

35. W. Santipach, M. L. Honig, in International Symposium on Information Theory, 2004. ISIT 2004. Proceedings. Asymptotic capacity of beamforming with limited feedback, (2004), p. 290. https://doi.org/10.1 109/ISIT.2004. 1365326

36. F. Ferrari, M. Zimmerling, L. Mottola, L. Thiele, in Proc. of the 10th ACM SenSys. Low-power wireless bus, (2012)

37. M. Zimmerling, L. Mottola, P. Kumar, F. Ferrari, L. Thiele, Adaptive real-time communication for wireless cyber-physical systems. ACM Trans. Cyber-Physical Syst. 1(2) (2017)

38. M. Grant, S. Boyd, CVX: Matlab software for disciplined convex programming, version 2.1 (2014). http://cvxr.com/cvx

39. M. Grant, S. Boyd, in Recent Advances in Learning and Control. Lecture Notes in Control and Information Sciences, ed. by V. Blondel, S. Boyd, and H. Kimura. Graph implementations for nonsmooth convex programs (Springer, New York City, 2008), pp. 95-110. http://stanford.edu/ boyd/ graph_dcp.html

40. J. Wang, D. P. Palomar, Worst-case robust MIMO transmission with imperfect channel knowledge. IEEE Trans. Signal Proc. 57(8), 3086-3100 (2009). https://doi.org/10.1109/TSP.2009.2021451

41. D. Goodman, N. Mandayam, Power control for wireless data. IEEE Pers. Commun. 7(2), 48-54 (2000). https://doi.org/10.1109/98.839331
42. F. Meshkati, H. V. Poor, S. C. Schwartz, N. B. Mandayam, An energy-efficient approach to power control and receiver design in wireless data networks. IEEE Trans. Commun. 53(11), 1885-1894 (2005). https://doi.org/10.1109/ TCOMM.2005.858695

43. F. Uhlig, A recurring theorem about pairs of quadratic forms and extensions: a survey. Linear Algebra Appl. 25, 219-237 (1979). https://doi. org/10.1016/0024-3795(79)90020-X

\section{Submit your manuscript to a SpringerOpen ${ }^{\circ}$ journal and benefit from:}

- Convenient online submission

- Rigorous peer review

- Open access: articles freely available online

- High visibility within the field

- Retaining the copyright to your article

Submit your next manuscript at $\boldsymbol{s p r i n g e r o p e n . c o m ~}$ 